\title{
Primary survey
}

Do you undertake emergency procedural sedation in non-fasted patients?

If you do, then the Preece and Benger (see page 254) provide reassurance that you are not putting your patients at significant risk. Their systematic review identified only one reported case of pulmonary aspiration during emergency procedural sedation, among 4657 adult and 17672 paediatric cases. The commentary by David Taylor (see page 253) highlights some limitations and calls for more research, but our best current evidence is that emergency procedural sedation in non-fasted patients is safe.

\section{Do senior doctors make a difference?}

Senior doctors are increasingly required to routinely review the decisions made by their junior colleagues. But is this just an expensive rubber-stamping exercise? The study by White and colleagues suggests not. Following senior review, 26 out of 165 proposed admissions were discharged and 10 out of 233 proposed discharge decisions were reversed and the patient admitted. Senior review also changed location of admission, speciality review and use of outpatients in a number of cases. One would hope that these changes to the management plan would be beneficial to patients and cost-effective, but proving this may be difficult (see page 262).

\section{Should we use MRI for suspected scaphoid fracture?}

Khalid et al report a series of 611 patients with scaphoid tenderness and no fracture on plain x-ray who underwent magnetic resonance imaging (MRI) as part of the standard protocol. The scans detected a lot of pathology and were abnormal in 342 cases $(56 \%)$, but does this improve patient outcome? We don't currently know what the best treatment for MRI-diagnosed scaphoid fracture is. Answering this question is an important research priority and would seem to be a pre-requisite to widespread use of MRI for diagnosis (see page 266).

\section{Too much pressure!}

Physicians and paramedics often feel the pressure when they perform prehospital or emergency department endotracheal intubation-and so, it appears, do the tracheal tube cuffs. Chopra and colleagues measured tracheal tube cuff pressures in 61 adults who were intubated prehospital or in the emergency department and found that $75 \%$ had a cuff pressure greater than $30 \mathrm{~cm}$ of water. This level of pressure carries the risk of tracheal mucosal necrosis (see page 270).

\section{Troponin measurement in suspected myocardial infarction and syncope}

The increasing sensitivity of troponin assays has led to controversy about the potential importance of low elevations of troponin. In this issue Body et al (see page 292) show that a troponin elevation below the functional sensitivity of the assay independently predicts an increased risk of subsequent adverse cardiac events. Adopting a lower cut-off for troponin would increase sensitivity for identifying subsequent adverse events at the expense of sacrificing specificity. The latter risk is illustrated by Lee et al (see page 302), who showed that only about half of patients with an initial troponin level that is low but above the $99^{\text {th }}$ centile, go on to have a troponin rise consistent with acute coronary syndrome on serial testing. Further research is required to identify the cut-off that maximises the benefits of identifying additional at risk cases without generating too many false positives requiring unnecessary investigation.

Meanwhile Reed and colleagues (see page 272) show that an elevated troponin is associated with an increased risk of adverse outcome in patients presenting with syncope. However, they counsel against routine measurement in patients with syncope because the diagnostic yield of positive troponin is low.

\section{What does the head injury leaflet} say?

Producing a leaflet is the easy part. Making sure that patients understand what it says is more difficult. MacDonald and colleagues analysed 45 head injury information leaflets used by hospitals in Scotland and found that less than $30 \%$ of the population are likely to understand more than $90 \%$ of the available head injury leaflets. Indeed it appeared that that $50 \%$ of the leaflets require an IQ of at least 100 to be understood. Presumably post-traumatic symptoms of headache, nausea, confusion and drowsiness don't help either (see page 279).

\section{In oxygen we trust}

A recent Cochrane review found that there was considerable uncertainty surrounding the effect of oxygen for patients with acute myocardial infarction and the potential for harm with a non-significant trend towards increased mortality. Under these circumstances a randomised trial would seem to be necessary. However, Burls et al found a distinct lack of equipoise among emergency department, cardiology and ambulance staff who responded to an online survey: $55 \%$ believed oxygen definitely or probably reduces the risk of death, while only $1.3 \%$ reported that they thought it may even increase the risk of death. Where has our faith in the healing power of oxygen come from? It doesn't seem to be based on the scientific evidence (see page 283).

\section{What makes a good ambulance service?}

Focussing on response times suggests that the only way to judge an ambulance service is by how fast the crews drive. Siriwardena and colleagues are looking at a different approach. In their first paper in this issue (see page 324) they discuss how recent developments in research support for ambulance trusts in England and Wales present the opportunity to establish a sound evidence base for what ambulance services should, and should not, do. In their second paper (see page 327) they describe how existing evidence is being used to develop clinical performance indicators for English ambulance services. Their laudable aims are to turn ambulance services into research capable organisations and to put quality of care at the heart of performance assessment. We wish them well.

\section{Which ballpoint?}

And finally, if you fancy yourself as a lifesaving hero using a ballpoint pen for an emergency surgical airway, make sure you carry a Baron retractable ballpoint or a BIC soft feel Jumbo. According to Owens and colleagues these are the only ballpoints that are up to the job (see page 317). 